\section{PATHOGENESIS OF ACUTE ISCHEMIC MITRAL REGURGITATION IN THREE DIMENSIONS}

Changes in the geometric and intravalvular relationships between subunits of the ovine mitral valve were measured before and after acute posterior wall myocardial infarction in three dimensions by means of sonomicrometry array localization. In 13 sheep, nine sonomicrometer transducers were attached around the mitral anulus and to the tip and base of each papillary muscle. Five additional transducers were placed on the epicardium. Snares were placed around three branches of the circumflex coronary artery. One to 2 weeks later, echocardiograms, dimension measurements, and left ventricular pressures were obtained before and after the coronary arteries were occluded. Data were obtained from seven sheep. Coronary occlusion infarcted $32 \%$ of the posterior left ventricle and produced 2 to $3+$ mitral regurgitation by Doppler color flow mapping. Multidimensional scaling of dimension measurements obtained from sonomicrometry transducers produced threedimensional spatial coordinates of each transducer location throughout the cardiac cycle before and after infarction and onset of mitral regurgitation. After posterior infarction, the mitral anulus enlarges asymmetrically along the posterior anulus, and the tip of the posterior papillary muscle moves $1.5 \pm 0.3 \mathrm{~mm}$ closer to the posterior commissure at end-systole. The posterior papillary muscle also elongates $1.9 \pm 0.3 \mathrm{~mm}$ at end-systole. The left ventricle enlarges asymmetrically and ventricular torsion along the long axis changes. The development of postinfarction mitral regurgitation appears to be the consequence of multiple small changes in ventricular shape and contractile deformation and in the spatial relationships of mitral valvular subunits. (J ThORAC CARDiovasc SuRg 1995;109:684-93)

Robert C. Gorman, MD (by invitation), James S. McCaughan, MD (by invitation), Mark B. Ratcliffe, MD (by invitation), Krishanu B. Gupta, PhD (by invitation), James T. Streicher, MSE (by invitation), Victor A. Ferrari, MD (by invitation), Martin G. St. John-Sutton, MBBS, FRCP (by invitation), Daniel K. Bogen, PhD, MD (by invitation), and L. Henry Edmunds, Jr., MD, Philadelphia, Pa.
In approximately one third of all patients with acute Imyocardial infarction a transitory apical systolic murmur develops within 2 weeks, ${ }^{1}$ and in many of these patients symptomatic mitral insufficiency subsequently develops months to years later. ${ }^{2}$ Between $19 \%$ and $30 \%$ of patients evaluated for coronary

From the Departments of Surgery, Medicine, and Bioengineering, Schools of Medicine and Engineering, University of Pennsylvania, Philadelphia, Pa.

Supported by grant HL 36308 from the National Heart, Lung, and Blood Institute, National Institutes of Health, Bethesda, Md.

Read at the Seventy-fourth Annual Meeting of The American Association for Thoracic Surgery, New York, N.Y., April 24-27, 1994.

Address for reprints: L. Henry Edmunds, Jr., MD, Department of Surgery, 4 Silverstein, Hospital of the University of Pennsylvania, 3400 Spruce St., Philadelphia, PA, 19104.

Copyright (C) 1995 by Mosby-Year Book, Inc.

$0022-5223 / 95 \$ 3.00+0 \quad 12 / 6 / 62471$ arterial disease have some degree of mitral insufficiency, ${ }^{3}$ and approximately one fifth of these patients have symptomatic regurgitation. ${ }^{3}$ Acute severe postinfarction mitral insufficiency with cardiogenic shock may be due to ruptured papillary muscle, ${ }^{4}$ but more often the papillary muscle is intact. ${ }^{4}$ The pathogenesis of mitral insufficiency resulting from myocardial infarction has intrigued investigators for years. ${ }^{5-8}$ Why does the valve leak when, with the exception of one papillary muscle, the structural components of the valve itself are not damaged?

The recent development of a sheep model of postinfarction mitral insufficiency ${ }^{7,9}$ and the technique of sonomicrometry array localization ${ }^{10}$ provide methods to address this question. The sheep model produces chronic or acute postinfarction mitral insufficiency by coronary arterial occlusion, as occurs in patients. No additional operation or injury is needed. Sonomicrometry array localization lo- 
cates tagged components of the mitral valve in three-dimensional space within the heart throughout the cardiac cycle. This article reports our first analysis of the geometric and intravalvular changes that occur in the ovine heart after acute severe postinfarction mitral insufficiency.

\section{Methods}

Initial instrumentation. In compliance with guidelines for humane care (NIH Publication No. 85-23, revised 1985), anesthesia was induced with thiopental sodium (10 to $15 \mathrm{mg} / \mathrm{kg}$ intravenously) in 13 Dorsett sheep (38 to 42 $\mathrm{kg}$ ). The animals were intubated and anesthetized with isoflurane $(1.5 \%$ to $2 \%)$ and oxygen. All animals received one dose of glycopyrrolate ( $0.4 \mathrm{mg}$ intravenously) and cefazolin ( $1 \mathrm{gm}$ intravenously) before the operation and one dose afterward. The surface electrocardiogram (ECG) and arterial blood pressure were monitored.

By means of sterile technique, a lateral left thoracotomy was performed and the heart was suspended in the pericardium. Five $3 \mathrm{~mm}, 5 \mathrm{MHz}$ ultrasonic dimension transducers (model LMT-530-PE, Crystal Biotech, Hopkinton, Mass.) were sutured to the epicardium at the left ventricular apex, posterior base, anterior free wall, anterior base, and on the left atrial appendage. Snares were placed around the second and third branches of the circumflex coronary artery and the posterior descending coronary artery. ${ }^{7}$

Heparin (15,000 U intravenously) was given and normothermic cardiopulmonary bypass was started. The perfusion circuit consisted of a $28 \mathrm{~F}$ wire-wrapped venous cannula (Medtronic Bio-Medicus, Eden Prairie, Minn.) placed into the right ventricle through the main pulmonary artery, a $14 \mathrm{~F}$ wire-wrapped arterial cannula in the left carotid artery, a centrifugal pump with precalibrated electromagnetic flowmeter (Medtronic Bio-Medicus), and a bubble oxygenator/heat exchanger (model H-1700, Bard Cardiopulmonary Division, Tewksbury, Mass.). After cardiopulmonary bypass was started, the heart was electrically fibrillated and the left atrial appendage was opened. Under direct visualization, $3 \mathrm{~mm} 5 \mathrm{MHz}$ ultrasonic dimension transducers (model LMT-530-PE) were attached to the tips and bases of both papillary muscles, to the mitral anulus at the anterior and posterior commissures, and over the midpoint of the anterior leaflet. Two additional transducers were attached to the posterior anulus at equidistant intervals. Transducers were attached by passing the wire through tissue and placing a polydiaxonone hemoclip (Ethicon, Inc., Somerville, N.J.) around the wire at the exit point. A total of nine intracardiac transducers were placed on mitral valve structures. Wires for the papillary muscle transducers were brought out through the left ventricular free wall and the annular transducer wires through the atriotomy. No wire crossed the plane of the mitral valve. After the atrium, was closed, the animals were defibrillated and weaned from cardiopulmonary bypass. The 14 color-coded transducer wires were placed in a subcutaneous polypropylene pouch and the wound was closed. Flunixin meglumine (100 mg intravenously) was given for analgesia (Fig. 1).
Data collection. Seven to 14 days after instrumentation, animals were reanesthetized with thiopental sodium, glycopyrrolate, and isoflurane. Under clean conditions a high-fidelity pressure transducer (SPC-350, Millar Instruments, Inc., Houston, Tex.) was passed into the left ventricle via a left carotid artery. The surface ECG and left ventricular pressure were monitored continuously (ES 1000, Gould Inc., Cleveland, Ohio). The wires of the ultrasonic transducers were exteriorized, sorted, and connected. Sonomicrometry was performed with a customized, digitally controlled sonomicrometer (J.T. Streicher, Philadelphia, Pa.) based on a standard eight-channel sonomicrometer (SONO-8, Physiologic Monitoring Systems Group, Durham, N.C.), a Motorola 68HC11-based microcontroller (New Micios Inc., Dallas, Tex.), and an IBM compatible personal computer (IT, Sperry Corp., Salt Lake City, Utah). In brief, individual transmitter and receiver circuits in the SONO-8 sonomicrometer are controlled by digital signals from the $68 \mathrm{HC} 11$ microcontroller. In this application the microcontroller sequentially activates a single transducer element followed by eight receiver elements to acquire eight intertransducer distances (data run). For each data run a different group of sonomicrometry transducer elements is manually connected to a single transmitter and eight receiver ports of the sonomicrometer. Analog left ventricular pressure, ECG, and valvular and ventricular dimension data were sampled at $100 \mathrm{~Hz}$ by a 16-channel, 12-bit analog-todigital converter board (Labmaster DMA, Scientific Solutions Inc., Solon, Ohio) in the Sperry IT personal computer and stored on the hard disk. Data from 16 separate runs were collected in a "gated" (by ECG) fashion to form a complete data set. The data set included dimensions, left ventricular pressure, and ECG. Each measurement was obtained with ventilation transiently arrested for 10 seconds at $5 \mathrm{~cm} \mathrm{H}_{2} \mathrm{O}$ airway pressure. Acquisition of a complete data set required 15 to 20 minutes and allowed measurement of all 91 intratransducer distances. ${ }^{10}$ After dimension measurements had been completed, transthoracic, short-axis echocardiograms were obtained (model 77020A, Hewlett-Packard, Inc., Santa Clara, Calif.). Transdiaphragmatic echocardiography for four-chamber views was performed through a clean midline laparotomy. Doppler color flow mapping was used to score the severity of mitral insufficiency.

The previously placed coronary arterial snares were tightened separately over 30 to 45 minutes and animals were allowed to achieve hemodynamic stability. The degree of mitral regurgitation was reassessed by color flow Doppler echocardiography. A second complete set of intratransducer dimension relationships was obtained after infarction. The sheep were killed with $1 \mathrm{gm}$ thiopental and $60 \mathrm{mEq}$ potassium chloride. The heart was removed without disturbing the transducers or wires. The position of each epicardial and endocardial transducer was verified. After the transducers had been removed, the left coronary artery was injected with triphenyltetrazolium chloride and sliced at $1 \mathrm{~cm}$ intervals. ${ }^{7}$ The size of the infarct was determined by planimetry. ${ }^{7}$

Data analysis. Customized, Windows-based software (K.B.G.) was used to analyze data. Cardiac cycle timing was based on the ECG and left ventricular pressure 


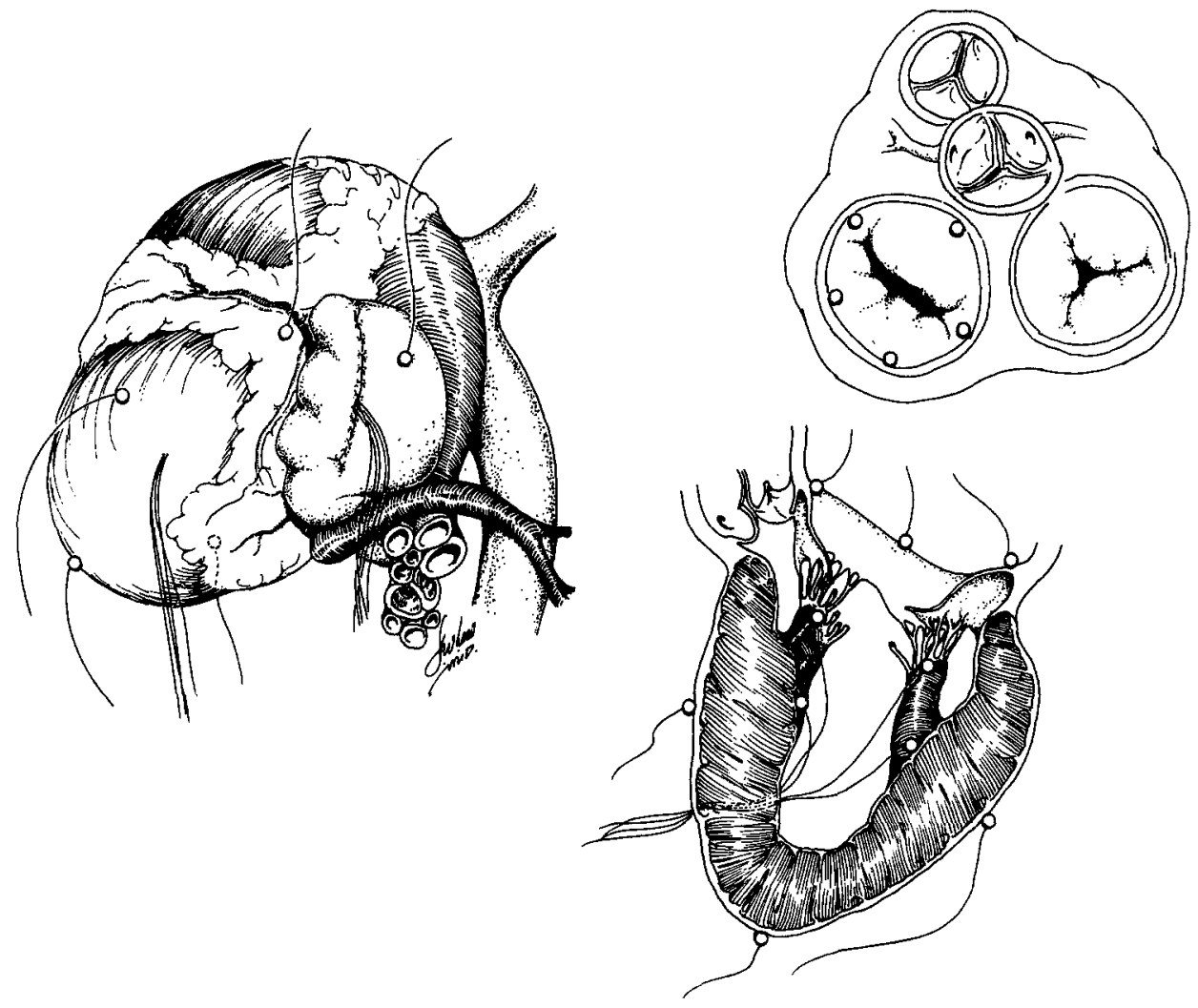

Fig. 1. Location of sonomicrometry transducers. Upper left views the heart from the left thoracotomy incision and shows transducer wires exiting the left ventricle and left atriotomy. The apical and four epicardial transducers are shown. Signals from the left atrial transducer were not analyzed. Upper right shows the five annular transducers; these wires exited the atrium. Lower right illustrates transducers attached to bases and tips of both papillary muscles and the apical, anterior, and posterior epicardial transducers.

tracing. End-diastole was defined as the R wave of the QRS. End-systole was defined as the point of maximum negative rate of $\mathrm{dp} / \mathrm{dt}$.

A previously described three-dimensional sonomicrometry array localization method was used to identify the relationship between all transducer positions in threedimensional space. ${ }^{10}$ In brief, the method uses all intertransducer distances obtained by sonomicrometry and the mathematical technique of multidimensional scaling to determine the spatial coordinates of each transducer. This imaging technique shows the positions of the transducers with respect to each other, but not with respect to a fixed frame of reference, such as the thoracic wall. Therefore, to view the three-dimensional image of the mitral apparatus (i.e., the set of transducer positions) and to compare data between sheep, we rotated it to a standard viewing orientation by the following procedure.

We first assumed that the five mitral annular transducers were approximately coplanar. A "best fit" plane was computed for the annular transducers. ${ }^{11}$ The image was then rotated so that the annular plane was parallel with the XY plane. Next, the image was translated to place the center of the anulus at the origin of the XYZ coordinate system. Finally, the image was rotated around the Z-axis to bring the anterior transducers into the $-Y$ (anterior) direction and the posterior transducers into the - $Y$ (posterior) direction.

\section{Results}

Of 13 sheep undergoing operation, three died at operation and two died or failed to recover fully within 10 days. Studies were started in eight sheep. One was found to have $1.5+$ mitral regurgitation before infarction and was excluded. Seven sheep completed the study. Ninety-six of the 98 implanted transducers in the seven sheep functioned satisfactorily; the two failures were in the same sheep.

Occlusion of the three coronary arteries infarcted approximately $32 \%$ of the left ventricular mass (Fig. 2), reduced systemic blood pressure, and produced mitral regurgitation (Table I). In contrast to the high-quality short-axis echocardiograms, long-axis 
Table I. Morphologic and hemodynamic variables

\begin{tabular}{lcc}
\hline & $\begin{array}{c}\text { Before } \\
\text { infarction }\end{array}$ & $\begin{array}{c}\text { After } \\
\text { infarction }\end{array}$ \\
\hline Size of infarction & - & $35 \pm 6.4$ \\
$\quad$ Echocardiogram (\% of circ) & - & $31.9 \pm 3$ \\
$\quad$ Planimetry (\% LV mass) & $103 \pm 7$ & $86 \pm 12 \dagger$ \\
LV systolic pressure (mm Hg) & $7 \pm 1.6$ & $19 \pm 4 \dagger$ \\
LV diastolic pressure (mm Hg) & 0 & $2+$ \\
Median Doppler score (0-4+ MR) & $0-1+$ & $1+-2.5+$ \\
Range (0-4+ MR) & $8.1 \pm 1.7$ & $9.4 \pm 2.1$ \\
Short-axis area &
\end{tabular}

Values are mean \pm standard error. $L V$, Left ventricular; circ, circumference; $M R$, mitral regurgitation.

*Three animals with hypotension after infarction had Doppler scores of $1+, 1.5+$, and $2+$. Three normotensive animals were scored $2.5+$ and one was scored $2+$.

$\dagger p<0.05$.

views did not fully image the apex and were more difficult to interpret (Fig. 3). However, the left atrium was clearly visualized. Mitral regurgitation was scored by Doppler color flow mapping of regurgitant jets actually observed according to established criteria, ${ }^{12,13}$ but it may have been underscored in hypotensive sheep. Three sheep were hypotensive (systolic left ventricular pressure 65 to $80 \mathrm{~mm} \mathrm{Hg}$ ) after infarction during Doppler color flow mapping.

Multidimensional scaling located all 14 transducer positions in three-dimensional space. By gating signals collected over 15 to 20 minutes while the animals were in hemodynamically stable condition, we were able to track movements of each transducer during the cardiac cycle. Each transducer inscribed an irregular loop that ended at the starting position. Actual movements of 14 transducers during systole before infarction are depicted in one sheep in Fig. 4. For these initial experiments, only positions at endsystole and end-diastole before and after infarction were analyzed; however, it is clear from Fig. 4 that relationships between tagged components of the valve vary considerably at other times during systole.

Positions of each transducer at end-systole before and after infarction as viewed in three orthogonal planes are depicted in Fig. 5. In panel $A$, an approximately sagittal view is shown. The plane of the mitral anulus is orthogonal to the plane of the graphic. The lengthening of the posterior papillary muscle after infarction at end-systole is clearly apparent (also see Table II). In the approximate coronal view (Fig. 5, $B$ ), the ventricle slightly lengthens after infarction and the axis of the anterior papillary muscle rotates clockwise (as viewed from atrium to ventricle). After infarction in the trans-

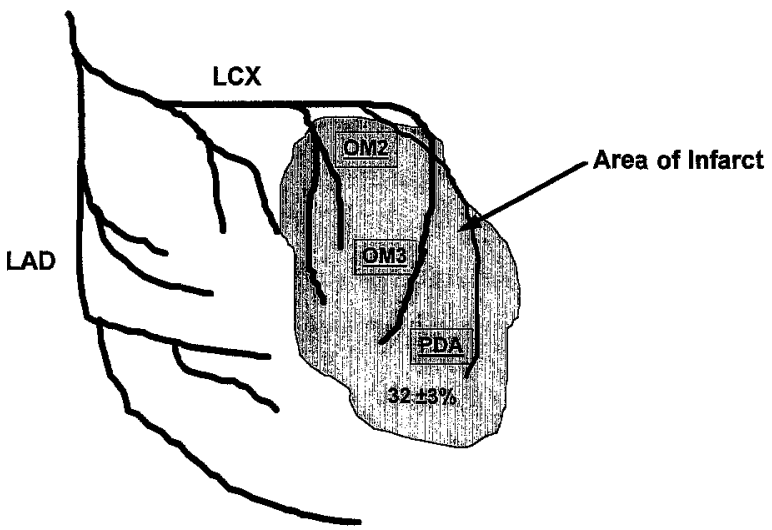

Fig. 2. Computer-generated drawing showing the location of the infarct produced by occlusion of the posterior descending (PDA) and obtuse marginal (OM2 and OM3) branches of the circumflex coronary artery. $L A D$, Left anterior descending.

verse view (Fig. 5, C), the anulus slightly enlarges and rotates counterclockwise; the ventricle and papillary muscles rotate clockwise.

Analysis of various distance changes between transducers in seven sheep further defines the deformation of the valve produced by infarction. The circumference of the anterior anulus does not appear to change (note only one transducer is attached to the anterior anulus; small changes in the arc subtended by the two chords cannot be excluded). However, the circumference of the posterior anulus significantly elongates after infarction in both diastole and systole (Table II). The elongation is not uniform across the posterior anulus (Table II). Transverse views in all seven animals confirm the asymmetric dilatation of the posterior anulus.

After infarction the posterior papillary muscle shortens slightly in systole but is elongated significantly as compared with its preinfarction end-systolic length. The tip of the posterior papillary muscle moves $1.5 \pm 0.3 \mathrm{~mm}$. closer to the posterior commissure at end-systole (Table II). The distance from the base of the posterior papillary muscle to the posterior commissure does not change at end-systole or end-diastole. Interrelationships between the base and tip of the anterior papillary muscle and the anterior commissure at end-systole and diastole are not changed by infarction.

The left ventricle dilates slightly after infarction and rotates around its long axis. The short-axis systolic cavity area increases (Table I) and the distance between the bases of the two papillary 


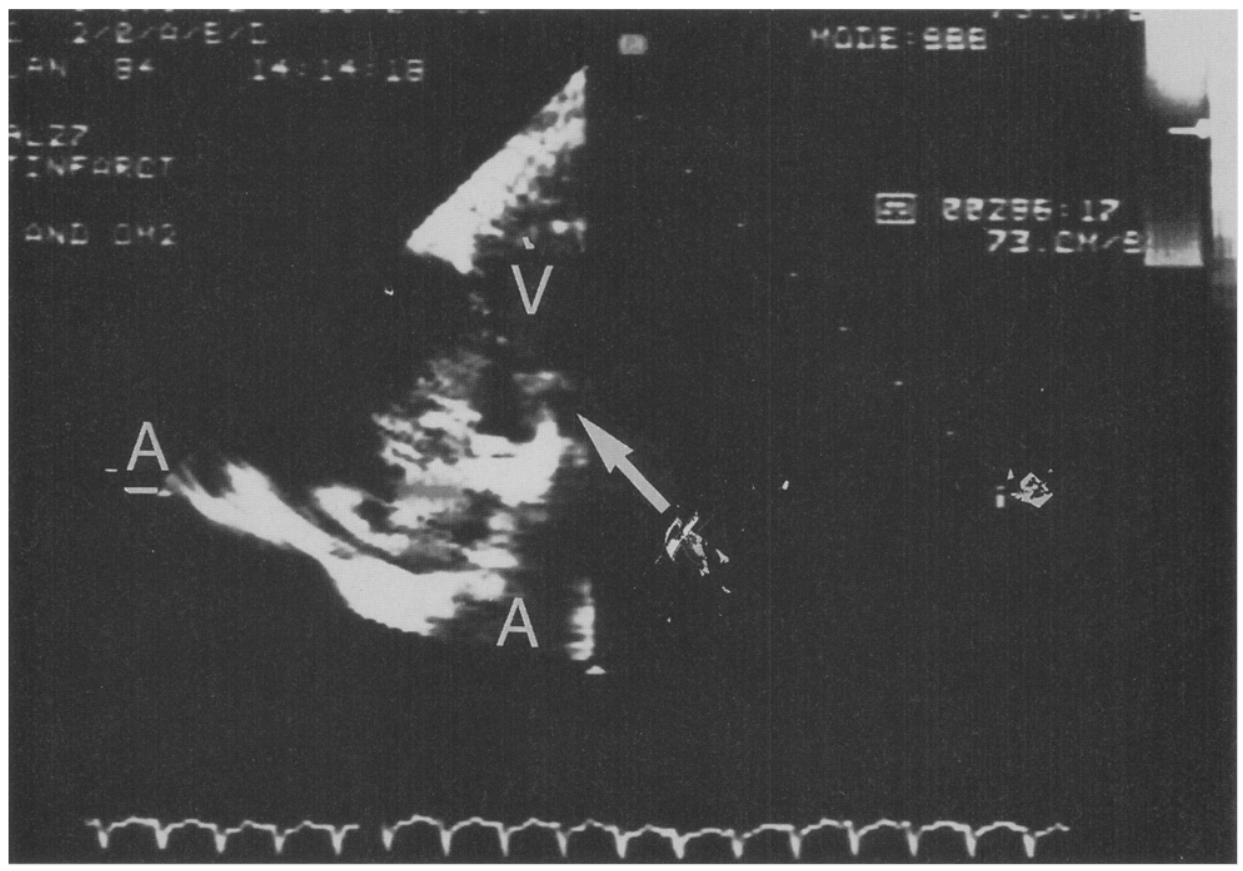

Fig. 3. Color flow Doppler echocardiogram from the apical long-axis orientation demonstrating moderate to severe $(2.5+)$ mitral regurgitation in the postinfarction state. $V$ denotes the left ventricle, $M$ designates the mitral valve plane, and the $A$ s depict the border of the left atrium. The light gray color within the atrium represents the jet of mitral regurgitation.

muscles increases significantly at end-systole but not at end-diastole (Table II). The enlargement is asymmetric. The distance between the base of the posterior papillary muscle and anterior commissure increases after infarction at both end-systole and end-diastole (Table II), but infarction does not significantly change the distance between the base of the anterior papillary muscle and posterior commissure (Table II). Transverse views in all animals show the opposite rotations of the anulus and ventricle at end-systole (Fig. 5, C).

\section{Discussion}

Postinfarction mitral regurgitation appears to be the consequence of several small changes in valvular subunits and the interrelationships between subunits. These changes deform the valve to cause the leak. No single change appears paramount. The anulus enlarges slightly and asymmetrically. The posterior papillary muscle lengthens, fails to contract, and the tip moves closer to the posterior commissure during systole. The ventricle also enlarges asymmetrically and rotates differently. In dynamic three-dimensional video depictions of selected, superimposed preinfarcted and postinfarcted hearts, the combination of asymmetric bulging and altered ventricular torsion appears to cause impressive deformation of the valve in some views. The infarct clearly alters the posterior papillary muscle and the size and shape of the mitral anulus: however, the immediate ventricular deformations produced by the infarct appear to have at least an equal, if not predominant, role in distorting the valve.

Sonomicrometry array localization with multidimensional scaling successfully locates tagged myocardial components in three-dimensional space and provides a method to track changes in the interrelationships of these components during the cardiac cycle over days or weeks. ${ }^{10}$ It is important to realize that sonomicrometry array localization calculates the coordinates of each functional transducer from measured distances from all other transducers. If all intertransducer distances could be measured simultaneously, the exact position of each transducer in relation to all others could be determined at all times throughout the cardiac cycle with minimal error. ${ }^{10}$ Present electronic technology requires some manual switching, and 15 to 20 minutes are needed to acquire all intertransducer distances. From these data, 10-second runs of high-quality signals are 


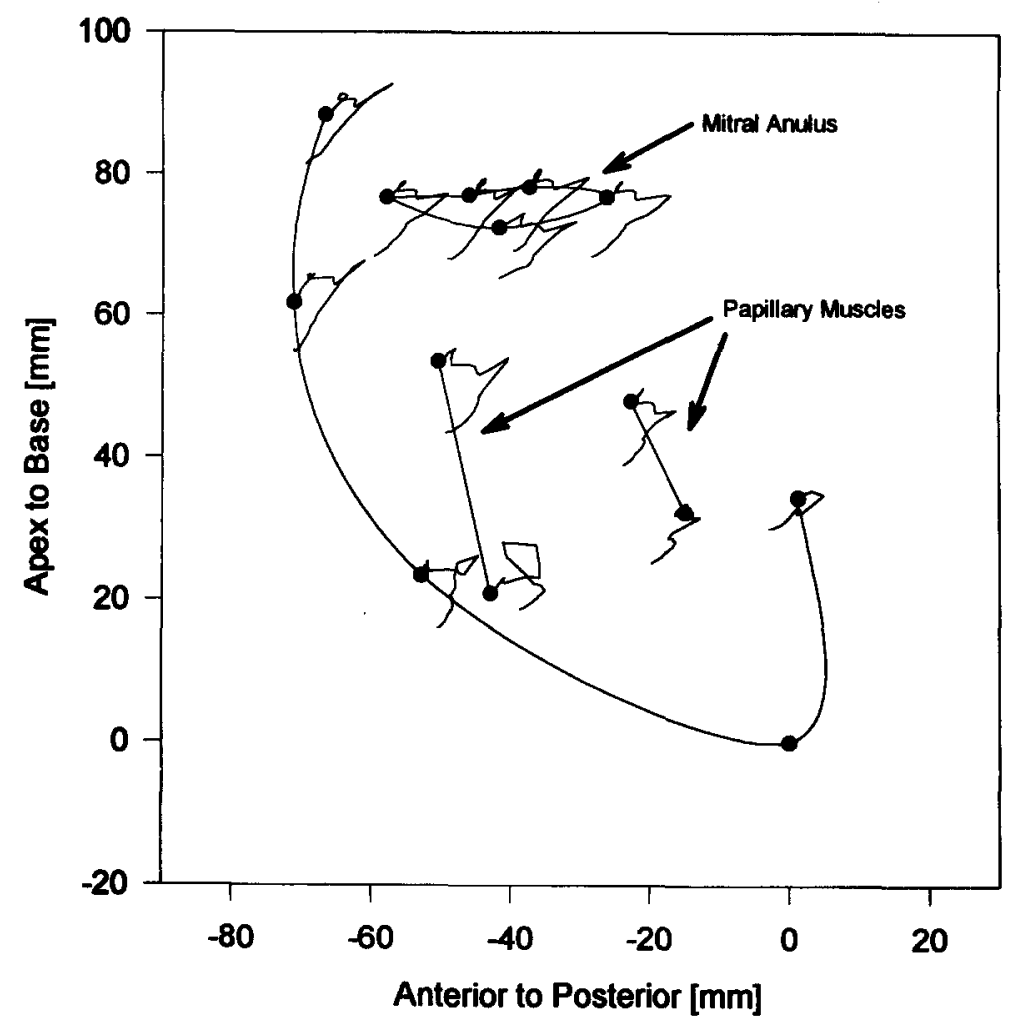

Fig. 4. Computer-generated graphic demonstrating the movement of each sonomicrometry transducer during systole before infarction in one sheep. The solid circle indicates the transducer position at the beginning of systole. The attached irregular line traces the movement of that transducer during systole. Transducers also move during diastole and end at the starting point. This graphic has been slightly tilted off plane and realigned to make the apex transducer the starting (reference) point.

selected to input into the multidimensional scaling program. Although animals remain in steady hemodynamic states during collection of data, physiologic variations in the interrelationships of the tagged mitral structures undoubtedly occur and probably introduce some random error in our measurements. Improved electronic switching, now being tested, acquires all intertransducer distances within 1 to 2 minutes; eventually, further electronic improvements should enable acquisition of all distances within a single heartbeat.

Our measurement technique allows us to observe changing distances within the heart and quantities that are measured from those changing distances: strain and torsion. We do not locate the transducers with respect to a coordinate system fixed to the skeleton of the sheep, and thus we are unable to determine movements of the whole heart, taken as a single body, that is, the rigid-body translation and rotation of the heart. However, we are able to describe relative motions of the mitral valve and papillary muscles, but not absolute motions of these structures with respect to fixed skeletal landmarks. Measurements of Lagrangian strain and torsion are correctly determined by our measurement technique and would not be improved by measurements of transducer positions taken with respect to a fixed coordinate system.

For comparisons of two-dimensional depictions of moving three-dimensional structures, a fixed observer point or "plane of reference" is needed. Our data do not provide a fixed reference plane; thus, for Figs. 4 and 5, we used the five mitral annular transducers to establish an internal plane of reference. It must be recognized that this internal plane probably moves a few degrees during the cardiac cycle as the mitral anulus warps, bends, and tilts. Although sonomicrometry array localization captures all three-dimensional movements of annular and other transducers during the heartbeat, small movements of the annular plane of reference introduce artifacts into orthogonal, 

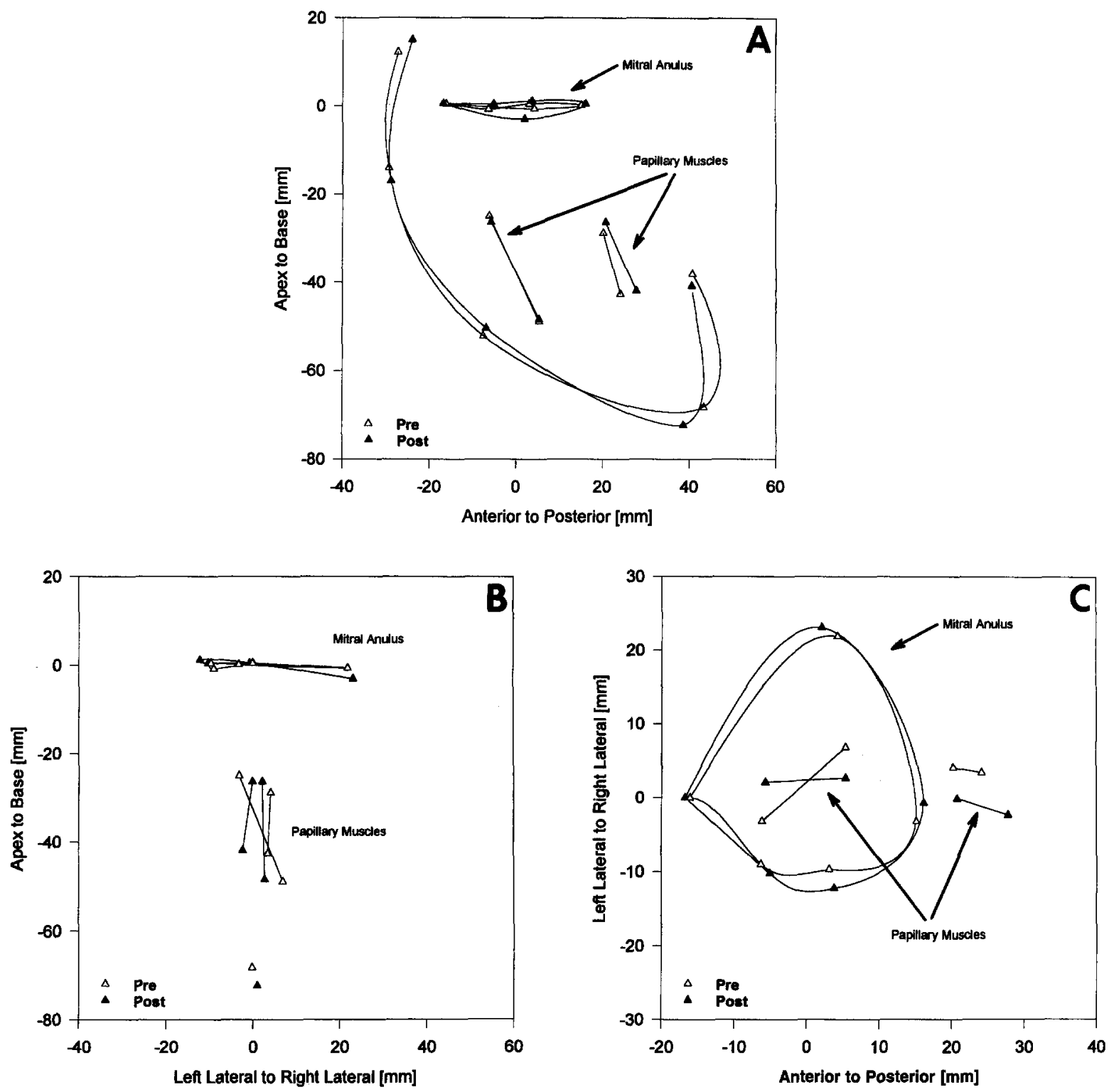

Fig. 5. Transducer positions before and after infarction shown in three orthogonal views at end-systole in one sheep. Open symbols indicate transducer positions before infarction; solid symbols indicate positions after infarction in all views. Lines between transducers were generated by cubic splines. ${ }^{25} \mathbf{A}$, In the left approximate sagittal view the anterior wall is left and posterior wall is right. The relationship between papillary muscle bases and the mitral anulus is apparent. $\mathbf{B}$, In this left to right lateral view (approximate coronal) the papillary muscles nearly overlap. Anterior is toward the right and apical transducers are shown. C, The transverse view is shown. Apical transducers are not shown but lie to the right of the figure.

two-dimensional depictions of transducer positions.

Between-sheep comparisons may be problematic because of anatomic and size variations and the fact that the sonomicrometry transducers are not placed in precisely the same locations in the mitral appa- ratus between sheep. These relativities increase standard deviations of between-sheep measurements. Fortunately, ovine cardiac and coronary arterial anatomy are surprisingly consistent, so that we are reasonably confident that the geometric distortions of the valve are similar between sheep. All of 
Table II. Selected dimension changes before and after infarction

\begin{tabular}{|c|c|c|c|c|c|c|}
\hline \multirow[b]{2}{*}{ Measurement } & \multicolumn{2}{|c|}{ Before infarction } & \multicolumn{2}{|c|}{ After infarction } & \multicolumn{2}{|c|}{ Difference } \\
\hline & $E D$ & $E S$ & $E D$ & $E S$ & $E D$ & $E S$ \\
\hline Total circumference (mm) & $90 \pm 2.9$ & $92.3 \pm 2.8$ & $94.1 \pm 2.8$ & $97.2 \pm 3.3$ & $4.1 \pm 0.7^{*}$ & $4.9 \pm 0.6 \dagger$ \\
\hline Circumference posterior anulus (mm) & $40.7 \pm 1.7$ & $40.8 \pm 1.6$ & $44.4 \pm 0.9$ & $44.3 \pm 0.7$ & $3.7 \pm 0.5 \dagger$ & $3.5 \pm 0.5 \dagger$ \\
\hline Circ Post Com to Pos Trans on Post anulus (mm) & $17.8 \pm 1.5$ & $17.9 \pm 1.0$ & $19.0 \pm 1.2$ & $19.5 \pm 1.0$ & $1.2 \pm 0.2 \dagger$ & $1.6 \pm 0.1 \dagger$ \\
\hline Circ Ant Com to Ant Trans on Post anulus (mm) & $11.2 \pm 1.4$ & $11.6 \pm 1.3$ & $12.6 \pm 1.2$ & $13.2 \pm 1.8$ & $1.4 \pm 0.2^{*}$ & $1.6 \pm 0.3^{*}$ \\
\hline Post Pap Base to Post Pap tip (mm) & $22.9 \pm 2.0$ & $21.2 \pm 2.0$ & $23.8 \pm 1.9$ & $23.1 \pm 1.0$ & $0.9 \pm 0.1 \dagger$ & $1.9 \pm 0.3 \dagger$ \\
\hline Post Pap tip to Post Com (mm) & $25.9 \pm 1.4$ & $26.7 \pm 1.4$ & $25.9 \pm 1.3$ & $25.2 \pm 1.0$ & $0 \pm 0.3$ & $-1.5 \pm 0.3 \dagger$ \\
\hline Ant Pap Base to Ant Pap tip (mm) & $29.7 \pm 5.6$ & $25.7 \pm 4.3$ & $29.2 \pm 4.8$ & $25.1 \pm 4.8$ & $-0.5 \pm 1.2$ & $-0.6 \pm 1.5$ \\
\hline Base Ant Pap to Base Post Pap (mm) & $33.0 \pm 3.2$ & $25.9 \pm 3.8$ & $32.5 \pm 3.4$ & $28.5 \pm 3.0$ & $1.5 \pm 0.2 \dagger$ & $2.6 \pm 0.4 \dagger$ \\
\hline Base Post Pap to Ant Com (mm) & $62.6 \pm 3.2$ & $60.5 \pm 3.1$ & $64.1 \pm 3.3$ & $62.9 \pm 3.0$ & $1.5 \pm 0.2 \dagger$ & $2.4 \pm 0.3 \dagger$ \\
\hline Base Ant Pap to Post Com (mm) & $57.2 \pm 2.6$ & $51.9 \pm 2.6$ & $56.9 \pm 2.6$ & $51.2 \pm 2.2$ & $-0.3 \pm 0.1$ & $-0.7 \pm 0.1$ \\
\hline
\end{tabular}

Circ, Circumferential; Post, posterior; Com, commissure; Ant, anterior; Trans, transducer; Pap, papillary muscle. Mean differences between end-diastole (ED) and end-systole $(E S) \pm$ standard error for all pairs reached $p<0.05$. Mean differences between preinfarction and postinfarction measurements of either ED or ES \pm standard error: ${ }^{*} p<0.05 ; \uparrow p<0.01$. The first four rows relate to the circumference of the anulus. Rows 3 and 4 indicate distances between either the posterior (row 3) or anterior commissure (row 4) and the adjacent transducer on the posterior anulus. The distances between the base of posterior papillary muscle and base of anterior papillary muscle (row 8) and between the base of posterior papillary muscle and the anterior commissure (row 9) after infarction indicate slight bulging of the posterior ventricular wall. In contrast, the distance between the base of the anterior papillary muscle and posterior commissure (row 10) does not change significantly after infarction. In sheep, the papillary muscles are part of the ventricular wall for approximately two thirds of their length and the free end to which chordae are attached represents only one third of the overall distance between base and tip. This anatomy and the bulging of the posterior ventricular wall after infarction may explain the apparent slight shortening of the infarcted posterior papillary muscle during systole (row 5).

the individual sheep show similar qualitative changes in the interrelationships of valve components as those shown in the figures for one sheep.

In the transverse views (see Fig. 5, C) showing papillary muscle and ventricular transducers orthogonal to the annular transducers, we observed clockwise rotation of the posterior papillary muscle transducers at end-systole after infarction. In most animals, some of the annular transducers rotate slightly counterclockwise as compared with their positions before infarction. Torsion or twist in the heart is defined as a wringing motion wherein two or more parts of the heart rotate in opposite directions around the same axis. Despite the limitations of our two-dimensional depictions of transducer positions after infarction, rotational movements of posterior papillary and annular transducers in opposite directions at end-systole indicate that infarction changes ventricular torsion during the cardiac contraction.

Sonomicrometry complements and expands serial, biplane, tantalum cineradiography that Ingels, ${ }^{14}$ Hansen, ${ }^{15}$ and their colleagues have used to study transplanted human hearts and the mitral valve in dogs. ${ }^{16,17}$ These investigators have pointed out the importance of ventricular twist during the cardiac cycle, particularly during different loading conditions. ${ }^{14}$ Both tantalum biplane cineradiography and sonomicrometry array localization can track changes in the location and movement of tagged myocardial islands during the cardiac cycle for days and weeks, and both methods have important advantages for experimental studies of ventricular remodeling. The tantalum method provides a permanent marker and acquisition of data at 60 frames per second; precision of measurements between transducers, relative ease of transducer identification and data analysis, and eventually rapid data acquisition are advantages of sonomicrometry array localization. ${ }^{10}$

In a recent study of posterior papillary muscle shortening after circumflex arterial occlusion, the Stanford group observed that posterior papillary muscle shortening, which normally is maximal during ejection, was delayed into the period of isovolumic relaxation. ${ }^{18} \mathrm{We}^{19}$ did not observe this delay in shortening after posterior infarction in sheep, and although we used slightly different definitions for end-systole and end-diastole, this difference is probably not sufficient to explain why our findings differ. For this question to be investigated satisfactorily, it will be necessary to examine the role of ventricular bulging after infarction (see legend, Table II), which confounds calculated volume measurements and probably affects ischemic papillary muscle lengths.

The size of the infarction in the present study was slightly less than that produced in our previous investigation, ${ }^{9}$ and the severity of mitral insufficiency was also less. Severe mitral regurgitation developed, however, and animals with steady albeit compromised hemodynamics survived long enough for us to obtain good dimensional data. The difference in infarct size between this and the previous study is probably due to the lack of concurrent 
thoracotomy in the present study and the addition of a large infarct near the end of a long operation in the previous investigation. ${ }^{9}$

The sheep model of ischemic mitral regurgitation mimics the human disease. Both acute and chronic mitral regurgitation can be produced in sheep. ${ }^{7,9}$ Some degree of mitral insufficiency is common after acute myocardial infarction, ${ }^{1-3}$ and in many patients valvular leakage persists and increases as the heart enlarges and remodels. This problem, which is often encountered clinically, has not been aggressively addressed therapeutically. ${ }^{20,21}$ Textbooks minimally cover the subject. Surgeons have not developed good operations for this condition, ${ }^{22-24}$ largely because the pathogenesis is poorly understood and because the valve looks normal in the flaccid, arrested heart. The combination of the sheep model and sonomicrometry array localization offers a better understanding of the pathogenesis of mitral regurgitation and a means to develop more effective and precise reparative operations.

We thank Nicolas Gikakis, Ted Plappert, Robert Helgans, and Andrew McMarlin for technical assistance.

\section{REFERENCES}

1. Gahl I, Sutton R, Pearson M, Caspari P, Lairet A, McDonald L. Mitral regurgitation in coronary disease. Br Heart J 1977;39:13-8.

2. Selzer A, Katayama E. Mitral regurgitation: clinical patterns, pathophysiology and natural history. Medicine 1972;51:337-66.

3. Hickey M StJ, Smith LR, Muhlbaier LH, et al. Current prognosis of ischemic mitral regurgitation. Circulation 1988;78(Suppl):I51-9.

4. Wei JY, Hutchins GM, Bulkley BH. Papillary muscle rupture and fatal acute myocardial infarction. Ann Intern Med 1979;90:149-53.

5. Tsakiris AG, Rastelli GC Amorim DD, Titus JL, Wood EH. Effect of experimental papillary muscle damage on mitral valve closure in intact anesthetized dogs. Mayo Clin Proc 1970;45:275-85.

6. Mittal AK, Langston M Jr, Cohn KE, Selzer A, Kerth WJ. Combined papillary muscle and left ventricular wall dysfunction as a cause of mitral regurgitation. Circulation 1971;44:174-80.

7. Llaneras MR, Nance ML, Streicher JT, et al. A large animal model of ischemic mitral regurgitation. Ann Thorac Surg [In press].

8. Kaul S, Spotnitz WD, Glasheen WP, Touchstone DA. Mechanism of ischemic mitral regurgitation. Circulation 1991;84:2167-80.

9. Llaneras MR, Nance ML, Streicher JT, et al. Pathogensis of ischemic mitral insufficiency. J THORAC CARDIOVASC SURg 1993;105:439-43.
10. Ratcliffe MB, Gupta KB, Streicher JT, Savage EB, Bogen DK, Edmunds LH Jr. Use of sonomicrometry and multidimensional scaling to determine the threedimensional coordinates of multiple cardiac locations. IEEE Trans Biomed Eng [In press].

11. Tampieri F. Newell method for computing the plane equation of a polygon. In Graphics Gens III, D, ed. New York: Academic Press, 1992:231-3.

12. Abbasi AS, Allen MW, Decristofaro D, Ungar I. Detection and estimation of the degree of mitral regurgitation by range gated pulsed Doppler echocardiography. Circulation 1980;61:143-7.

13. Spain MG, Smith MD, Grayburn PA, Harlamert EA, Demaria AN. Quantitative assessment of mitral regurgitation by Doppler color flow imaging: angiographic and hemodynamic correlation. J Am Coll Cardiol 1989;13:585-90.

14. Ingels NB Jr, Hansen DE, Daughters GT II, Stinson EB, Alderman EL, Miller DC. Relation between longitudinal, circumferential, and oblique shortening and torsional deformation in the left ventricle of the transplanted human heart. Circ Res 1989;64:915-27.

15. Hansen DE, Daughters GT II, Alderman EL, Ingels $\mathrm{NB}$, Stinson EB, Miller DC. Effect of volume loading, pressure loading, and inotrophic stimulation on left ventricular torsion in humans. Circulation 1991;83: 1315-26.

16. DeAnda A Jr, Moon MR, Yun KL, Daughters GT II, Ingels NB Jr, Miller DC. The immediate effects of cardiac surgery on left ventricular torsional dynamics. Circulation 1993;88(Suppl 1):1435.

17. Castro LJ, Moon MR, Rayhill SC, et al. Annuloplasty with flexible or rigid ring does not alter left ventricular systolic performance, energetics, or ventricular-arterial coupling in conscious closed-chest dogs. J THORAC CARDIOVASC SuRg 1993;105:643-59.

18. Rayhill SC, Daughters GT II, Castro LJ, et al. Dynamics of normal and ischemic canine papillary muscles. Circ Res 1994;74:1179-87.

19. Gorman RC, McCaughan JS, Ratcliffe MB, Gupta $\mathrm{KB}$, Guy TS, Edmunds LH Jr. A three-dimensional analysis of papillary muscle spatial relationships in acute postinfarction mitral insufficiency. Surg Forum 1994;45:330-4.

20. Karp RB, Mills N, Edmunds LH Jr. Coronary artery bypass grafting in the presence of valvular disease. Circulation 1989;79(Suppl 1):I182-4.

21. Arcidi JM Jr, Hebeler RF, Craver JM, Jones EL, Hatcher CR Jr, Guyton RA. Treatment of moderate mitral regurgitation and coronary disease by coronary bypass alone. J Thorac Cardiovasc Surg 1988;95: 951-9.

22. Hendren WG, Memec JJ, Lytle BW, et al. Mitral valve repair for ischemic mitral insufficiency. Ann Thorac Surg 1991;52:1246-52.

23. Rankin JS, Fenely MP, Hickey MS, et al. A clinical 
comparison of mitral valve repair versus valve replacement in ischemic mitral regurgitation. J THORAC Cardiovasc Surg 1988;95:165-77.

24. Cohn LH. Surgical treatment of ischemic mitral regurgitation by repair and replacement. In: Ischemic mitral incompetence. Vetter HO, Hetzer R, Schmutzler H, eds. New York: Springer-Verlag, 1991:179-86.

25. Ferziger JH. Numeric methods for engineering applications. New York: John Wiley, 1981:1-23.

\section{Discussion}

Dr. William D. Spotnitz (Charlottesville, Va.). At the University of Virginia, we created a canine model of acute ischemic mitral regurgitation in which there were two separate, independently regulatable, and adjustable circulations to the papillary muscles themselves and to the rest of the ventricular myocardium. As described in a 1991 issue of Circulation, we could induce reversible ischemia of the papillary muscles alone or of the rest of the myocardium and then measure the degree of mitral regurgitation.

In this preparation, parameters that measured left ventricular systolic function, such as rate of left ventricular pressure rise, aortic root pressure, and the degree of circumferential myocardial wall thickening, showed excellent correlations with the amount of mitral regurgitation. However, parameters of anterior and posterior papillary muscle function, specifically thickening as measured echocardiographically, showed poor correlation with the degree of mitral regurgitation.

The mitral regurgitation closely correlated with decreased global ventricular function and incomplete mitral leaflet closure. With papillary muscle dysfunction alone, there was no significant mitral regurgitation and no mitral leaffet prolapse.

My question in your model is whether you could evaluate the relative independent contributions of global ventricular, papillary muscle, and annular function to ischemic mitral regurgitation.

Dr. Gorman. I cannot tell you exactly how each one of these factors contributes to the regurgitant valve. All we know is that the sum total of these factors lead to a massively regurgitant valve. We have not been able to dissect out exactly how much each one contributes to the mitral insufficiency.

Dr. Radu Deac (Tirgu-Mures, Romania). This excellent study proves once more the concept that the mitral valve acts in concert with the other components of the left side of the heart. Morphometric studies of the mitral valve show that for a mean orifice of $7 \mathrm{~cm}^{2}$ the surface of the leaflet is double, approximately $14 \mathrm{~cm}^{2}$, to be able to safely close this orifice. Systolic contraction of the posterior part of the mitral anulus, demonstrated in this study, reduces the orifice area to be closed by the leaflets. Together with a continuity to the papillary muscle, this mechanism involves the mitral valve in the mechanics of the left ventricle. The study showed also that the geometry and dynamics of the left ventricle are important for the normal function of the mitral valve.

This study has a profound implication for the future of mitral valve surgery. The results of mitral valve surgery can be improved by maintaining the systolic constriction of the mitral valve and continuity to the papillary muscle.

Dr. Robert W. M. Frater (Bronx, N.Y.). We have taken a very simple approach to this subject matter, and I think the answer as to whether we are right may be in your data.

Two things happen between systole and diastole: In diastole the anulus dilates and the posterior left ventricular wall elongates; in systole they both shorten. We have looked at the echoes, obtained measurements in our patients with posterior ventricular infarctions and mitral insufficiency, and evaluated the distance between the papillary muscle and the anulus. Whereas in the normal person that distance shortens in systole, in the patient with a posterior ventricular infarct it fails to shorten; it holds the papillary muscle down away from the anulus and keeps the cusps apart at their tips.

Annuloplasty can be used to compensate for that. Thus, if you do an annuloplasty in these cases, and that is all we do in acute mitral insufficiency, you can correct it.

With your elegant system, can you tell me whether the distance from the base of the papillary muscle to the anulus either elongated or failed to shorten during systole in the infarcted cases?

Dr. Gorman. The distance from the base of the posterior papillary muscle to the mitral anulus initially elongates slightly during isovolumic contraction and then decreases rapidly with ejection. After infarction, in this model, we did not notice a change in that cord length.

Dr. Frater. Are you saying that in both the infarcted segment and the noninfected segment they both behaved normally?

Dr. Gorman. That is correct. The relationship between the base of the posterior papillary muscle and the annular plane is unchanged after infarction. 